\title{
Grow: visualising nature at nanoscale
}

\author{
Erica Seccombe \\ Australian National University \\ School of Art, Building 105, Ellery Crescent, Acton ACT Australia \\ Erica.Seccombe@anu.edu.au
}

\begin{abstract}
Animating time and space: scientific virtual modelling, microscopy time-lapse and the fifth dimension. As a visual artist I am exploring methods of contemporary scientific research and digital technologies that create new ways of visualising virtual volumetric data of dynamic (or kinetic) microscopic systems. My current research project is an investigation of the aesthetic possibilities of computational extension of vision through the process of dynamic X-ray micro-computed tomography (micro-CT). With this science I am attempting to capture in $4 D(3 D+$ time) microscopy, the transformation of agricultural seeds as they germinate, from embryo to first leaf stage. I am visualising these dynamic data-sets in in a custom designed volume exploration and presentation tool Drishti.
\end{abstract}

Volumetric data, animation, virtual modelling, time-lapse, tomography, $X$-ray, microscopy art, science.

\section{INTRODUCTION}

Perception is a master of space in the same measure in which action is a master of time.

Henri Bergson, Matter and Memory, 1912.

The growing leverage of computational power allows computer-simulated objects or environments to become essential tools within scientific and technological disciplines. Virtual modelling technology aims to present objects or phenomena in a manner identical to their natural counterpart. The tradition of scientific modelling provides a logical and objective way to represent objects and processes while communicating theories and concepts. Modelling functions to visualise, manipulate and simulate objects in silico are greatly extended, particularly if the data is acquired from microscopy or X-ray technologies that capture material normally invisible to the human eye. The additional capability to move and relocate the viewpoint of the observer in relation to the position of the object within the virtual space, whether in real-time or as animated sequences, increases the opportunity for the human imagination to make new determinations, observations or predictions.

As a visual artist, not a scientist, I have begun to investigate this experience of observation and analysis through the visualisation and animation of 3D data from dynamic microscopic systems. Contemporary technologies provide innovative processes for observing microscopic material, and this increasing comparative scale offers a unique perspective from which to reflect as observers, creating new meanings across time and circumstance. However, where scientific microscopy seeks evidence to support a hypothesis, reducing the object down to its very elements for examination, I am interested in how this abstraction and scale in an animated $3 D$ virtual environment can be experienced as art. As an artist I do not want to create purely didactic works. My challenge is to find a way of making meaningful works of art with this same scientific methodology, while creating new narratives that can extend beyond the technology and the original object.

GROW: visualising nature at nanoscale is the working title I have given my research project, in which I am investigating the aesthetic possibilities of computational extension of vision through the process of dynamic X-ray computed tomography microscopy (micro-CT). My interdisciplinary project combines both data acquisition and the visualisation of this data. For this purpose I am attempting to capture in 3D time-lapse the germinating process of agricultural seeds, from embryo to first leaf stage. I am then animating the resulting data by using custom designed volumetric exploration software. My intention is to create work that will allow an audience to experience seed propagation at a scale enlarged 


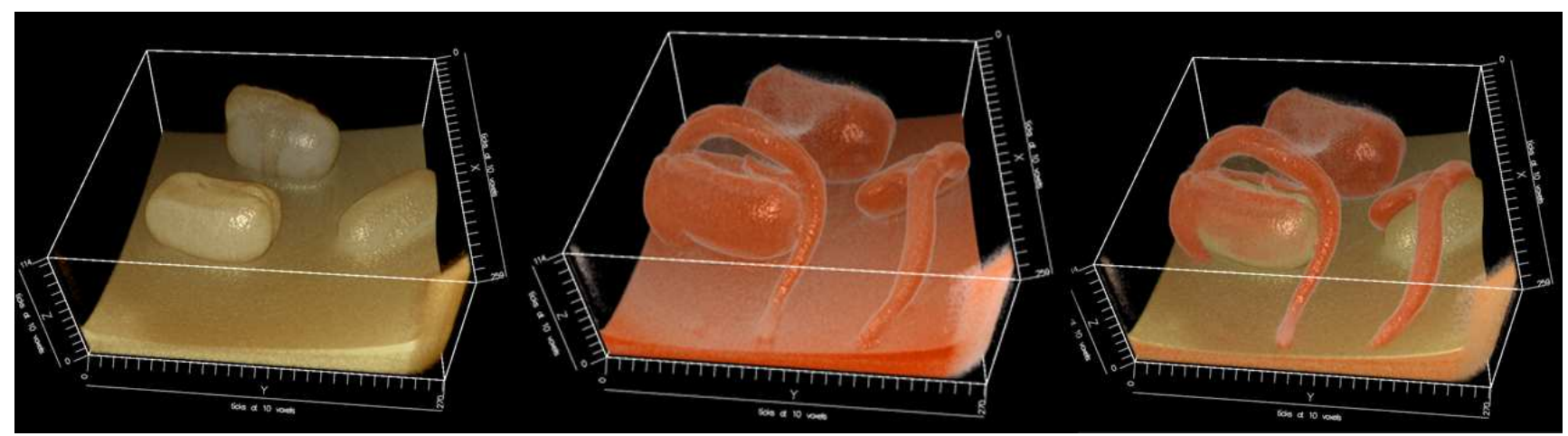

Figure 1: Volumetric time-datasets of seeds sprouting showing the beginning (yellow) and end point (orange) of shoot growth, the third set an overlay of both datasets. Imaged in Drishti, E.Seccombe \& Dr.A Limaye, 2011, ANU.

well beyond the natural proportion of the original, natural process. The premise of propagating crop seeds, rather than other varieties such as Australian natives, is to consider contemporary questions surrounding the comparative values between perceived natural and artificially cultivated food sources, particularly in context of the current concerns for global food security within a sustainable and natural environment.

At the time of writing this paper I am still in the early experimental stages of this project and the capabilities of the technology are rapidly evolving. To describe this work-in-progress in perspective of my creative practice, I will explain the interdisciplinary nature of the project and my artistic premise alongside the scientific technology in which it is situated. So as to discuss the multidimensional properties of animating volumetric data with the addition of time, I will consider the exploratory virtual object as three-dimensional and the inclusion of discrete kinetic moments over time as the fourth dimension. As I intend to make work to be experienced by an audience, I will also contemplate the phenomenological potential for sensation, meaning and affect in art as an additional fifth dimension.

Researchers at the ANU Department of Applied Mathematics' XCT Facility are conducting pioneering research in the field of dynamic X-ray computed tomography microscopy. The Department includes physicists, chemists and mathematicians engaged in research of an experimental and theoretical nature. They are recognised for groundbreaking research into processes involving the computational imaging of complex materials and networks and their results have challenged theoretical knowledge of the structure of matter, including the established understanding of evolution.

For example, in 2005 a 380 million year old gogonasus fossil was discovered by Tim Senden as part of a team of leading Australian paleontologists. He then scanned and visualised the fossil using micro-CT, enabling the team to observe the interior structure of this rare fossil without destroying the original material. The resulting virtual model of the ancient fish skull has revealed sub-layers of sensory nodes within the cranial cavity and snout. This information has paved the way for new insights into the evolutionary link between fish and land animals, as they left the water to walk, smell and breath air (Long et al, 2006). By applying the same methods these researchers also discovered the first definite evidence of a placental birth in a Late Devonian placoderm fossil, proving that vertebrate copulation began some 200 million years than previously estimated (Long et al, 2006).

\section{THE THIRD DIMENSION AS VOLUMETRIC DATA}

As a non-scientist I play no part in the business of implementing algorithims for segmentation of 3D datasets, but I think it is essential to understand how the data is acquired and visualised in order to plan and develop work while utilising this technology.

It is important to clarify that the $3 D$ data I am investigating and utilising are not derived from fragmentary evidence or simulated and rendered through mesh-framing techniques as you might expect with computer generated imagery. Instead the objects I am exploring are the algorithmic recreation of actual material data collected from high definition micro-computed technologies. These datasets are virtual replications of an object; each volumetric pixel representing its real-life particle. The visualisation technology gives the observer the most precise virtual model possible of the original microscopic object, simulating the texture and material density of both the internal and exterior structure. 
In the last decade the ANU Department of Applied Math's range of optical technology and software has greatly extended their experimental capabilities and their XCT Facility offers the highest fidelity computer tomography available. It is supported by APAC, Australia's largest public supercomputer centre which maintains an enormous volume (several petabytes) of archival storage at the ANU. The 3D fabric of material, such as rock or bone, encodes the mechanical properties of the object and the complex interplay between structure, form and material composition can only be solved with a supercomputer. The portrayal of dense datasets is not a simple task and the Department aims to balance visual density with information content, recognising that this articulation requires guidance by a visual sense in combination with computational power.

In 2004 the Department built and implemented a prototype micro-CT with the ability to image materials of various density and size, from a maximum of five-centimeter samples to a resolution of one micron. Computed tomography (CT or CAT scans) developed mainly from the needs of medical $X$-ray imaging and is generally recognised by familiar detailed images of body parts in thin slices. Advances in industrial 3D micro-CT have enabled an object to be X-rayed around a 360 degree rotation on a fixed axis. This complete tomographic scan is then recreated through computer processing of digital geometry to generate a three dimensional image of the internal and surface areas of a static object. One single dataset may contain up to twenty gigabytes of information, resulting in high-resolution 3D volumetric data represented by voxels or points mapped within $x, y$ and $z$ coordinates. This data is synthesised and then reconstructed by segmentation, which is the interpretation of this $3 \mathrm{D}$ image by a computer. These phases differentiate the various aspects of data such as solids, liquids and air, as a process of improving the clarity of the data. The more dense the original sample, the longer the process takes.

As an end-user I have become independent in the acquisition and manipulation of these datasets through a unique purpose-designed volume exploration software Drishti, meaning 'insight' or 'vision' in Sanskrit. So named by its creator $\mathrm{Dr}$ Ajay Lamaye, Drishti has been developed in-house specifically to assist end-users to visualise volumetric data from a wide range of scientific disciplines and outputs, and it is continually upgraded to adapt to emergent technologies. Ajay works closely with the XCT Facility to progress the software capabilities with research demand. The central idea for Drishti is to enable scientists to explore volumetric datasets as well as create interesting and engaging presentations with visual

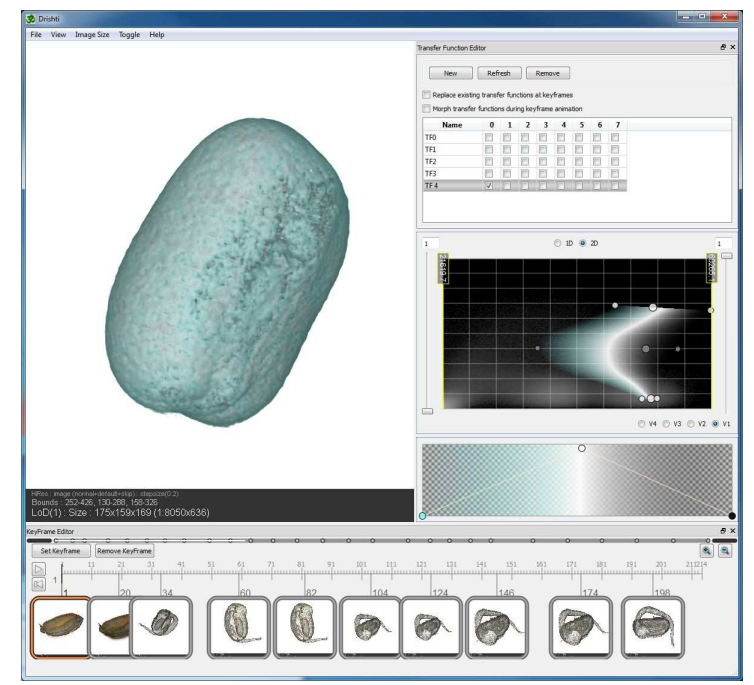

Figure 2: Detail of Drishti window. Volumetric datasets of a seed in the process of sprouting being imaged and animated in Drishti, E.Seccombe, 2011, ANU.

data. While analysis of material data is a primary focus for researchers, Drishti's premise in visualising datasets acknowledges that the information is equally important in conveying understanding to a research community or to nonscientists.

Importing raw datasets into Drishti enables the user to identify the essential material by trimming a clean sub-volume for rendering. Datasets acquired through X-ray tomography microscopy have no colour definition when they appear as virtual objects in the main window. Unlike other meshframed and surface rendered computer graphic programs, the density of the 3D object can be determined by using the program's transfer functions and histogram panel. The user can then distinguish between the material density of the surface and look through into the sub-layers by a process of elimination or enhancement, and by controlling the transparency and opacity of selected data. To enhance the object or prepare it for particular observation and analysis, the software has a wide range of choice in manipulating the data through colour, cropping, filters, clipping, lighting and contrast. The level of technicality depends entirely on the requirements of the project and the complexity of the dataset.

In the virtual space of Drishti's main window the 3D object can be examined from any angle. The observer's position and distance from the object are operated in real-time using mouse and keyboard control. Viewpoints are saved onto a keyframe editor as discrete moments. The length and frequency of the frames in-between each viewpoint can then be determined by adjusting the number of incremental steps. The speed and the length of the animation are determined by the frequency of key frames per second. The moving path of the object 
from one moment to the next is automated either by using the key frame editor or by calculating the exact position, angle and degree of rotational angles required. For example, 0 to 360 degrees will make one full rotation and a direct central path from front to back will create an iconic fly-through of the object. The resulting animation files are compatible with most generic movie platforms, as well as for stereoscopic and 3D interactive tools.

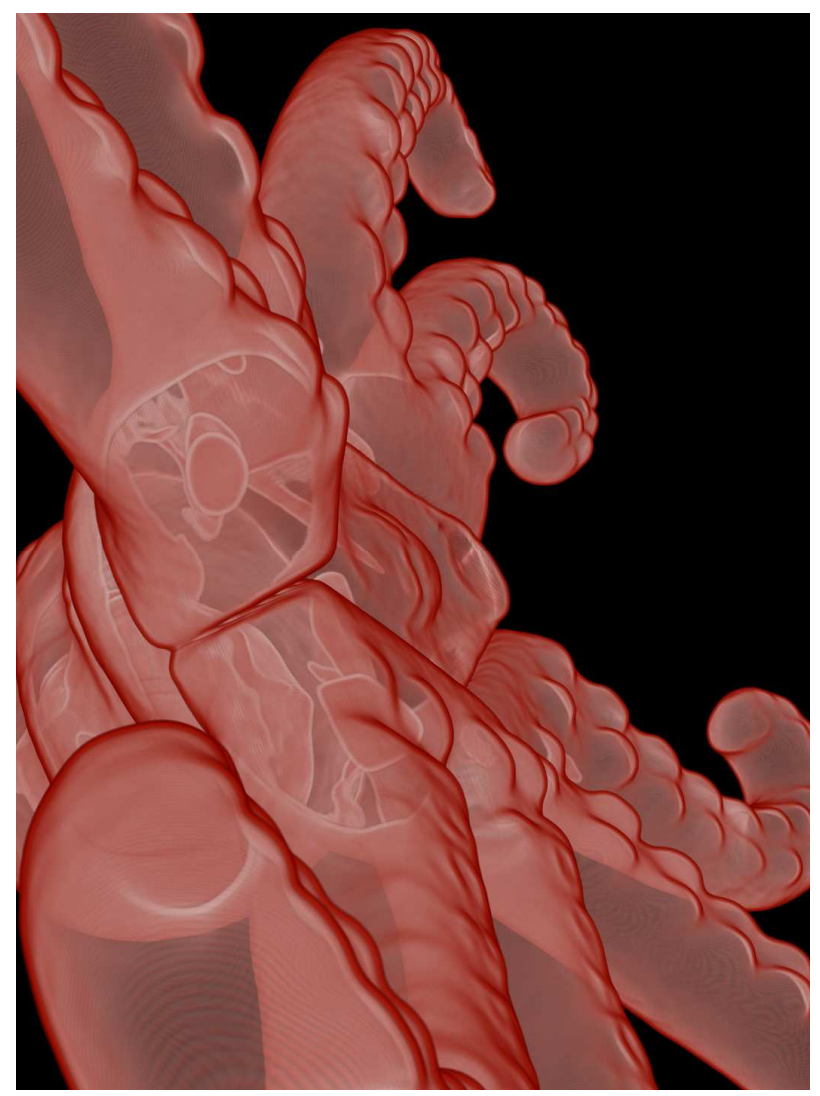

Figure 3: Erica Seccombe, Nanoplastica (detail), 2008, 3-channel digital projection installation.

Since its inception in 2004, Drishti's user interface has improved considerably, but as a continual user I have observed that visualising true volumetric data is also an acquired skill for scientists unless they have prior experience of programming and other digital media. The ability to image a dataset is not a straightforward procedure if you are unfamiliar with interpreting volumes though histograms or navigating around a virtual 3D environment. The first time I used Drishti in 2006 to visualise volumentric datasets I found it unlike any commercial animation or graphics package I have ever used. As a non-scientist I experienced a steep learning curve, first learning how to recognise my data through histograms, how to control volumentric data in a virtual space, and then to create visually smooth animations through what was then a very frustrating and time consuming process.
My first completed body of work using virtual 3D objects animated in Drishti resulted in the exhibition Nanoplastica at Canberra Contemporary Art Space in 2008. For this first work I sought ways to emulate scientific research as a means of examining issues of visualisation and replication of the natural world, touching on concepts drawn from the sciences such as evolution, genetics, microbes and nanotechnology. In the XCT Facility we scanned a selection of miniature plastic toys representing mammals, insects and marine animals. Collectables from a brand of novelty chocolate, each toy is no bigger than three centimetres and designed to be assembled by joining several parts together. I chose these objects because of their potential to translate interestingly through microCT. Rather than using specimens of real insects or invertebrates, my deliberate use of these readymade objects juxtaposed the established understanding of what is natural and artificial, while acknowledging the history of artists' employment of quotidian objects as cultural commentary.

Visualising these datasets with Drishti, I created new meanings for these toys that extend well beyond the original reading of the object. Having lost their real-life materiality, the virtual internal structure is revealed beneath a translucent, incandescent skin. To position the viewer in relation to the objects, I placed the creatures at the foreground of the centre screen so as to appear enormous and near, the interior structures appearing in and out of view as they slowly rotate clockwise. I installed Nanoplastica by projecting three choreographed sequences of these animations side by side, from ceiling to floor along an 8 by 20 meter wall, so that as an installation it dominated the entire gallery space. Playing on a

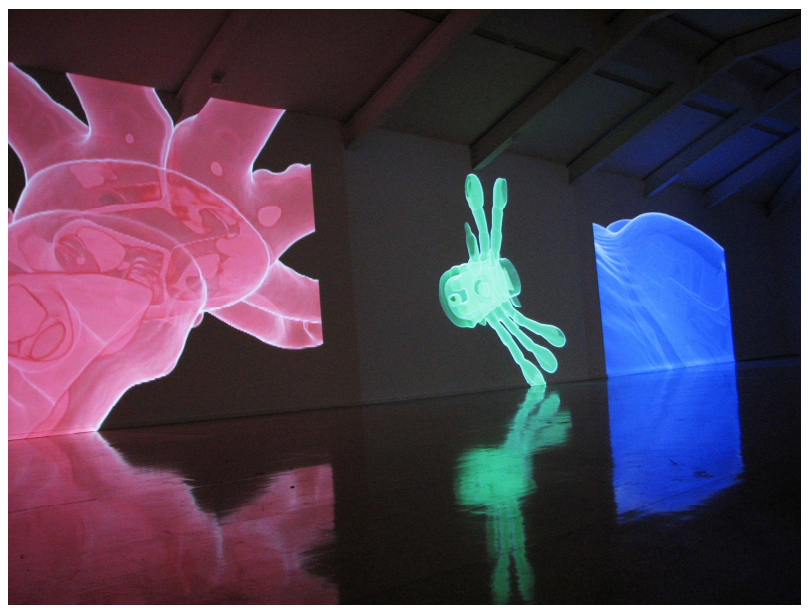

Figure 4: Erica Seccombe, Nanoplastica, 2008, 3-channel digital projection installation. Installed at Canberra Contemporary Artspace, 2008, Canberra, Australia. site-dimensions; CCAS main gallery $20390 \mathrm{~cm}$ wide $x 8750 \mathrm{~cm}$ depth, with a ceiling height of $4450 \mathrm{~cm}$ from floor to the beginning of the roof beams 
continuous loop, each projection varied in length so that no same combinations of images were repeated for any one viewer. Taken out of context, transformed and enlarged beyond their original size these virtual creatures begin to realise a life of their own.

Alluding to microscopy, Nanoplastica pays homage to scientific endeavour in a historical context, referring to early scientific representations and categorisation of nature, ultimately setting out to challenge our modern-day knowledge and perspective of the natural world. I also wanted to share with the audience the same fascination I had for these virtual objects, and as I had been making the work in a fairly isolated environment I was uncertain about the reception of the work. What I gained from this exhibition was how the audience responded to their emotional and physical experience of this work. The slow rotation of the transparent and seductively coloured objects were considered mesmerising and the scale allencompassing. People questioned the curiously familiar objects, wondering if they were observing animalcules, infusoria or futuristic nano-robots.

Even though these animations were not processed stereoscopically, I was also encouraged by the positive feedback about the virtual experience of the 3D objects, which varied between adults. It was remarkable to watch small children who would, quite dangerously, run and slam into the wall as the creatures slowly turned; it is as if they believed they could pass through the wall and into the object itself. This realisation about the emotional response to this work motivated me to consider the potential for subjective experience in future projects. This inspired me to pursue my new project GROW and investigate the additional dimension of time.

\section{THE FOURTH DIMENSION AS VOLUMETRIC DATA PLUS TIME}

Another description of Dynamic X-ray microscopy tomography, or 3D data with time-lapse is 4D micro-CT, indicating that the fourth dimension is the added property of time $(3 D+$ time $)$.

Until recently, the capabilities of micro-CT were limited to imaging tiny samples of static objects, such as bone or rock. My plastic toys were just at the limit of the scanning diameter. Since my first residency in 2006, the XCT Facility has greatly extended its reach through refinement and improvement of resolution and decreased acquisition time. The current researchers have built and installed a new prototype micro-CT instrument that captures both the volume and length of the object; the axis rotates 360 degrees while moving upward on a helical rotation, increasing the capacity for sample size. With these new capabilities they have embarked on an experimental project of 4D micro-CT. The acquisition of $3 D$ data in cinematographic mode and the analysis of time evolution in microscopic 3D systems, such as water wicking through fibre or flowing through rock, was only marginally feasible until recently. This group in the Department has now patented a novel method for the rapid acquisition of kinetic 3D datasets.

Financial investment from the mining industry occupies a majority of the Department's research into capturing data from dynamic systems, such as the viability of extracting gas and fluids from rock. I was not convinced that I could create work from rock samples, even though they are interesting to observe in Drishti. I wanted to find a subject that continued my interest in artificial representations of nature so I proposed that I would be interested in looking at sprouting seeds. Neither I nor the Department had ever worked with organic matter before in this context and we encountered a range of challenges including how to grow seeds in an waterless, irradiated environment for extended periods of time. There are also many variables such as the uneven growth rate of seeds or whether the seeds will grow in soil or other material.

At present I have been working with 16 datasets from a 9 hour time-lapse capture of two mung beans and a radish seed. I have singled out the mung bean and created several short animations that show it sprout until the root is about two centimetres long. In these initial stages I have been defining the potential for animation and how the next series of micro-CT scans might be customised to influence future data acquisition of sprouting seeds. The added dimension of time to $3 D$ data has massively increased the computational requirements in both the size of the data and the methods of sequencing these volumes though animation. Generating up to eight billion volumetric data points, each possessing nine properties, into a

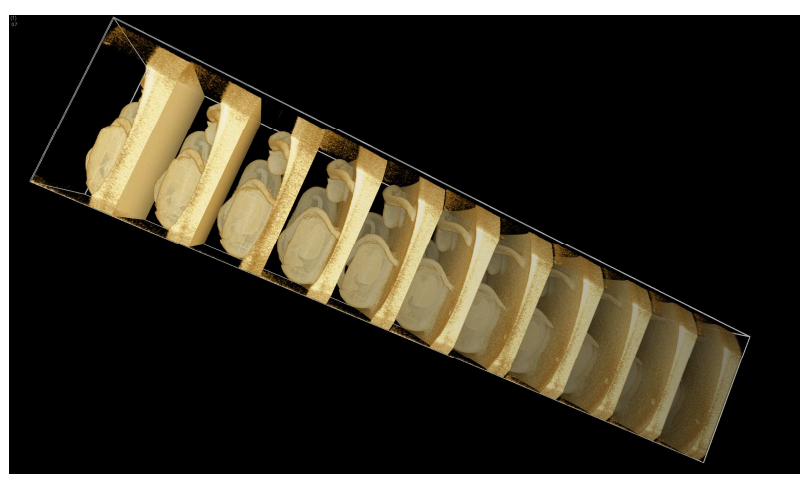

Figure 5: Detail of a volumetric time-stacks or 3D 'film strip' of seeds sprouting. Imaged in Maha Drishti, Dr A.Limay, 2011, ANU 
meaningful animation has required further consideration and program development for Drishti. In the last month Ajay has met these challenges of visualising dynamic micro-CT with a new software version called Maha Drishti, Maha meaning massive. With Maha Drishti, Ajay has been able to animate larger 4D datasets and this unprecedented dynamic data capture and its subsequent visualisation are still being perfected. My sprouting seeds have been captured in discreet moments, not in one entire 4D sequence as much of the newer rock samples have been. To create a visually smooth transition from one $3 D$ dataset to another along a timeline has required Ajay to interpolate the datasets so as to give the illusion of movement. The process of visualising my seeds and other 4D data has revealed new ways of looking at the keyframes. For example, as I began to write this paper, Ajay has visualised for the first time an assemblage of dynamic volumes in a volumetric stack. Analogous to the frames in filmstrips, each step or discrete moment appears before the last. The difference is that the stack is also an object that can be rotated and examined in a virtual space. I can view the stages of seeds sprouting as if it were a sculpture; turning it around from every angle to observe the sequential pattern of growth.

The conventional technique of time-lapse photography, to control the frequency of movement over a period of time, captures phenomena that in real-time is imperceptible to the human eye. Quickened or slowed, time lapse provides a single position for the observer, even it is from the viewpoint of the microbe or a bug. 4D micro-CT extends our vision further, enabling a rare insight into a dynamic microscopic system that until recently was completely inaccessible to us. Reconstructed and visualised as a virtual form, we can now experience seeds at the point of germination with simultaneous and multiple perspectives from both the exterior and interior.

By animating the dynamic datasets in Drishti, I can see the delicate central architecture of the mung bean through the fuzzy outer skin of the testa, or seed coat. As the bean swells drawing up moisture, it transforms inside as the first stirrings of the cotyledon, the embryonic leaf, creates curious bubbles in a central chamber. The unpredictable and organic nature of the data has also changed the way I first envisaged the appearance of the work. The material data of the beans are not crisp like the plastic animals for Nanoplastica, which provided clean-cut shapes and surfaces. The beans are spongy and the various stages of growth differ in density and contrast. It would be impossible to recreate this ambiguous texture and translucent skin using mesh frames. These are true virtual seeds suspended in an artificial space. With this technology we can experience this moment of transformation, looking out through the delicate casing as the plumale, the root, lengthens above, searching for a suitable place to anchor in the surrounding virtual space.

\section{THE FIFTH DIMENSION AS SENSATION, MEANING AND AFFECT}

I want to replicate for an audience the sensation I experienced when I first saw these exciting glimpses of virtual seeds sprouting. I envisage an exhibition of animated digital projections that enables an audience to fly through the virtual seeds and experience the internal structure as it germinates, travelling along the new shoot as it grows. By opening up an unseen world and further extending the experience to another physical dimension, these dynamic virtual models present multiple possibilities for reflection, discussion and interpretation. This relational quality of observation has led $m e$ to contemplate the emotional experience for scientists, past and present, who witness or discover something that no one else has ever seen or experienced before. To enable a similar experience to occur as part of this new work, I am looking into a variety of new media technologies and installation techniques, including perhaps interactive platforms or stereoscopic filters to make GROW a unique experience.

My response to the growing phenomena of genetic engineering and plant phenomic technologies is to capture in 4D the kinetic process of propagating economic seeds - such as mung bean, corn, sunflower, soy and wheat. My interest in microscopy visualisation has led me to consider the science of biotechnology and the debate concerning the future of agriculture. As agriculture is a founding component of civilisation, the genetic modification of food is neither a simple nor a single subject. I am beginning to explore how the phenomena and mythology of agriculture are understood, including ethics, biogenetics, history, tradition, socio-economics, trade networks, industry production, and biodiversity.

The increase in our reliance on technology to mass-produce food is driven by demand and consumption. Over-population creates more complex issues such as the escalation of foodinsecurity, urbanisation, carbon-constraints and climate change. In 2010 the Australian Commonwealth Scientific and Industrial Research Organisation (CSIRO) warned that current crop yields will need to be doubled by 2050 to feed a growing global population, predicting that to overcome the predominant challenges of reduced natural resources and the threat of potential 
hazards, the forthcoming agricultural revolution will need to be directed by new technologies and genetic improvements (Clark, 2009). I am curious about the growing negative reaction to genetically modified food when we seem unable to find ethical solutions to a sustainable society.

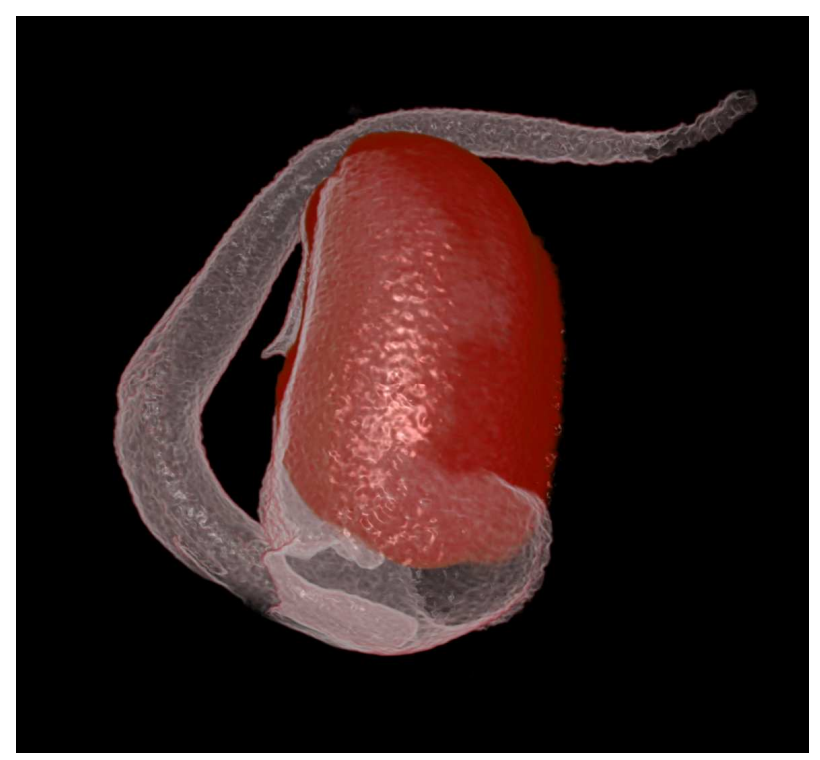

Figure 6: Overlaid volumetric datasets of a seed in the process of sprouting. Imaged in Drishti, E.Seccombe, 2011, ANU.

My interest for these debates and topics informs the basis of my project, but my intention is not to generate a body of work that represents scientific evidence or idealises notions of nature. I hope to achieve a body of work that provides a more subjective experience of a virtual seed germinating. As part of my research project I am in the process of understanding how the concept of phenomenology, as a study of structures of perception as a meaningful experience from an individual's point of view, can work in the context of contemporary art. As part of this investigation I have been looking at the work of artists who use methods of locating the body within the selfconscious experience of or about an object. In particular I have been interested in understanding how these artists expand the sensory properties of the aesthetic experience and purposely engage the viewer with alternative perspectives.

For example, the contemporary Icelandic space and light artist Olafur Eliasson employs strategies in his work to create a subjective experience of the interior and exterior spaces of his objects and installations. He has described the additional individual experience of his work as the fifth dimension, and connects this to the concept of temporality and greater relativity in an understanding of the third or fourth dimensions (Grynsztejn, 2007). Phenomenology has been a major source of inspiration to Eliasson who first became interested in the subject as an art student in the 1980s as it offered him a means for understanding subjectivity and ways of engaging with his surroundings. Eliasson notes that for previous generations phenomenology was used more as a formula for categorising experience, and he warns that phenomenology should not be justified as a kind of truth, but instead should be considered, 'as a tool for negotiating and reevaluating the environment - and this can pave the way for a more causal relationship with our surroundings.' (Grynsztejn, 2007).

Eliasson's work is temporal and atmospheric, and many of his installations are accessible and engaging. He invites his audience to become part of the work, as part of the experience. Eliasson is preoccupied with phenomena derived from what are referred to as classical elements and forces of nature, but believes that 'nature is a product of civilisation,' and that as we carry with us our experiences of life, socialisation and civilisation are embodied within each of us. Therefore regardless of what circumstance we happen to find ourselves in, our view of nature is changed the moment we look at it. (Koerner von Gustorf \& Morais, 2006).

For his installation, The Weather Project, commissioned for the Tate Modern's Turbine Hall in 2003, Eliasson transformed the space into a vast, tonal landscape saturated with mist and a giant globe of radiant light. Representing the sun and sky, it is as if he were able to suspend time permanently in that moment when the sun sets or rises on the horizon, perhaps a metaphor for the last day on Earth or the dawn of a new era. Viewers were able to observe themselves within the work, their reflections captured high above in a in a suspended mirrored ceiling. Rather than making claims about climate change, Eliasson instead takes the universal subject of weather to explore ideas around the circumstantial experience and representation of our surroundings. Eliasson's view is that phenomenology, '...offers an inquisitive, explorative approach to the world that allows for multiple perspectives on artworks, subjectivity, and experience. (Koerner von Gustorf \& Morais, 2006).

In the context of Eliasson's approach to subjectivity and natural phenomena, two recently published papers, one by Sarah Engelmann and another by Lesley Duxbury, respond to phenomenologist methods in contemporary art practice as a model for engaging with the uncertainties of environmental change. Both Duxbury and Engelmann describe society as being bound to inaction toward the complex problems of the environment because of an ingrained rhetoric in our relationship to nature. While we are bound to nature as we are to life, there is a perceived 
division between the purposes of civilisation and the preservation of a natural environment. To reevaluate our relationship to nature, both writers propose that we can shift our perception by understanding the subjective experience of natural phenomena in the work of artists such as Eliasson. This alternative experience can offer new ways comprehending our circumstance and enable a response toward positive action.

In her essay, 'Breaking the frame: Olafur Eliasson's art, Merleau-Ponty's phenomenology and the rhetoric of eco-activism', Englemann proposes a new model for environmental protest. She writes that by observing the methodology of contemporary artists such as Eliasson, we have the potential to break from conventional views of the environment and to set new 'global paradigms, leaning toward sustainability and long-term interaction between society and ecosystems.' (Englemann, 2011). In 'A Change in the Climate: New Interpretations and Perceptions of Climate Change through Artistic Interventions and Representations', Duxbury discusses the polarisation of society toward the hard facts for global warming. Identifying that in the face of potentially cataclysmic events scientific evidence may be working against encouraging social change in society, she suggests that we could also be looking at works of art that have the potential to engage society with nature, 'in emotional and experiential ways.' (Duxbury 2010). She argues that the methodology of artists like Eliasson whose practice is more than just a purely imaginative or aesthetic activity can now be considered, 'integral to meaningful communication between humans and the changing world.' (Duxbury 2010).

By considering these two essays and the work of artists such as Olafur Eliasson, many questions are raised about my installation of virtual germinating seeds and how a future audience might respond to it. I envisage a final body of work that will allow an audience to engage in a virtual experience of seed propagation at a scale that is enlarged well beyond the proportion of the original, natural process. While the object of the virtual seed is a starting point, the activity of witnessing or interacting with the previously invisible moment of germination will hopefully create a varied range of individual experiences; particularly when a majority of us are already far removed from the physical process of cultivating food.

At this stage of my project I am unable to write about the actual subjective experiences for GROW: visualising nature at nanoscale, as the work is still several years in the making. I don't intend provide solutions or answers in relation to genetically modified crops, a sustainable future or the preservation of bio-diversity in the natural environment. The potential of my own project GROW to create a subjective experience, as an additional fifth dimension, remains a concept to contemplate. Rather than finding an outcome, the purpose of this paper is to discuss the direction of the project, and to describe some aspects of the physical and thinking processes of my creative practice.

I see my project as having dimensions that contain numerous components moving along a series of interlinked axes through time and space. Exploring the interconnectedness of the subjects and the objects of my investigation opens up the possibility for new contextual meanings. By employing a combination of X-ray, microscopy, computational vision technologies, visual art and philosophy, digital media and traditional time-lapse and animation techniques, I acknowledge that each separate component in itself symbolises an historical route of human endeavour. Over the centuries these paths have overlapped and interfused to create new events, new technologies and new applications, shaping our world as we know it. In the act of engaging an audience, I will be extending this experience, hopefully generating new understandings and consequential exchanges.

The final narrative of GROW will ultimately be determined by the limitations of technology available to me, both in the acquisition of data, the visualisation and the equipment for its installation as a work of art. By transcending conventional images of seeds germinating, and pushing this work beyond a purely scientific interpretation of data, I hope that it will succeed in merging science, technology, art and culture in an innovative way. Importantly, I would like to preserve that moment of awe and discovery of seeing something ordinary, such as the sprouting of a seed, in a new way and for the first time.

\section{ACKNOWLEDGMENTS}

My interdisciplinary, practice-led research project GROW: visualising nature at nanoscale, conducted as a $\mathrm{PhD}$ candidate in the Australian National University (ANU School of Art), has been made possible because of the unique and collaborative relationship I have been fortunate to establish with researchers in the Department of Applied Mathematics at the ANU School of Physical Sciences and Engineering. My acquisition and use of $3 \mathrm{D}$ datasets and this recent investigation into dynamic 3D systems is facilitated by Professor Tim Senden and his colleagues in the Department's XCT Facility, and Vizlab at the ANU Supercomputer Facility. My first artistic residency with the Department took place in 2006. Since then I have been privileged to benefit from the ongoing 
support and encouragement of the Department's staff.

ANU School of Engineering and Physical Sciences Department of Applied Mathematics

\section{XCT Laboratory}

Professor Tim Senden, Dr Andrew Kingston, Dr Glenn Myers , Dr Trond Varslot \& Dr Michael Turner

\section{ANU Supercomputer Facility}

Dr Ajay Limaye creator of Drishti

VizLab

Mr Stuart Ramsden \& Mr Drew Whitehouse

\section{REFERENCES}

Bergson, H. (1912) Matter and Memory, London, Allen \& Unwin, New York, MacMillan (revised edition,. 1929)

Duxbury, L (2010) A Change in the Climate: New Interpretations and Perceptions of Climate Change through Artistic Interventions and Representations,.., Weather, Climate \& Society, Volume 2, 2010, 294-299

Long, J.A., Young, G.C., Holland, T., Senden, T.J. \& Fitzgerald, E.M. (2006) An exceptional Devonian fish from Australia sheds light on tetrapod origins', $\begin{array}{llll}\text { Nature } & 444 & \text { (7116): }\end{array}$ doi10.1038/nature05243

Engelmann, S. (2011) Breaking the frame: Olafur Eliasson's art, Merleau-Ponty's phenomenology and the rhetoric of eco-activism, , art\&education http://www.artandeducation.net/paper/breaking-theframe-olafur-eliasson's-art-merleau-ponty'sphenomenology-and-the-rhetoric-of-eco-activism/ (retrieved 10 May 2011)

Koerner von Gustorf, O. \& Morais, M. (2006) Cultural history, not natural history: an interview with Olafur Eliasson. Translation: Scrima, A., Berlin Deutche Bank magazine, http://www.dbartmag.com/archiv/06/e/magazin-

eliassoninterview.html, (retrieved 10/11/2011)

Grynsztejn, M., ed. (2007) Take your time: in conversation, with Olafur Eliasson and Robert Irwin, in Take your time: Olafur Eliasson, exhibition catalogue, San Francisco Museum of Modern Art, 52.

Clark, M. (2009) Sustainable Agriculture: Feeding the World, , adapted transcript of a speech delivered to the Science and Technology in Society Forum, Japan, 6 October 2009. http://www.csiro.au/science/ Sustainable-Agriculture-Feeding-the-World.html, (retrieved 8/09/2010) 